\title{
Oxyphilic Adenocarcinoma
}

National Cancer Institute

\section{Source}

National Cancer Institute. Oxyphilic Adenocarcinoma. NCI Thesaurus. Code C3679.

An adenocarcinoma characterized by the presence of large malig nant epithelial cells with abundant granular eosinophilic cytoplasm (oncocytes). Representative examples include thyroid gland oncocytic follicular carcinoma, oncocytic breast carcinoma, and salivary gland oncocytic carcinoma. 